\title{
Anisotropy and non-linearity of absorption of intensive IR light by free electrons in germanium
}

\author{
V.M. Vasetskii, V.A. Ignatenko, V.N. Poroshin \\ Institute of Physics, NAS of Ukraine, 46, prospect Nauky, 03028 Kyiv, Ukraine \\ Fax: (38 044) 525-15-89, e-mail: poroshin@iop.kiev.ua
}

\begin{abstract}
The anisotropy and non-linearity of absorption of the intensive $\mathrm{CO}_{2}$ laser radiation by free electrons in germanium has been found. The effect is caused by redistribution of electrons among equivalent valleys occurring due to non-equal heating electrons in the valleys which have different orientation in regard to the electric field of the light wave.
\end{abstract}

Keywords: multivalley semiconductors, light absorption by free carriers, anisotropy, non-linearity, heating carriers, intervalley redistribution.

Manuscript received 05.07.05; accepted for publication 25.10.05.

\section{Introduction}

Light absorption by free carriers in the cubic multivalley semiconductors (Ge, $\mathrm{Si}$ ) is isotropic. The anisotropy of absorption arises with violation of a uniform distribution of electrons over the equivalent valleys because of lowering the symmetry of their distribution in the wavevector space. This phenomenon was observed in $n$-Ge under elastic directional deformation of a crystal as well as in the course of heating carriers by electric field $[1,2]$.

Redistribution of carriers among the valleys may be caused by a light wave itself in the case when its intensity is sufficiently high [3]. Earlier we have found experimentally the birefringence [4] and the four-wave interaction (FWI) [5] for the $\mathrm{CO}_{2}$ laser radiation in $\mathrm{Ge}$ crystals with the carrier concentration $N=5 \cdot 10^{16} \mathrm{~cm}^{-3}$ at the temperatures 300 and $77 \mathrm{~K}$. These effects were related with the refraction index variation caused by intervalley redistribution of electrons in a light wave field.

The main cause of redistribution consists in different heating electrons during light absorption by them in different valleys. Therefore, this redistribution exists only in the case when the light wave electric field $\mathbf{E}$ is directed asymmetrically in regard to the long valley axes in a crystal. At the same time, the extent of the intervalley redistribution depends on the light intensity. Therefore, the light absorption by electrons must be both anisotropic and non-linear. A theoretical analysis of the anisotropy and non-linearity of light absorption by free electrons caused by the intervalley redistribution was carried out in [6]. In this paper, we report on experimental demonstration and study of this phenomenon in Ge crystals under irradiation by intense IR light with the wavelength $10.6 \mu \mathrm{m}$.

We studied the reciprocal transmission $\Lambda^{-1}=I_{\text {in }} / I_{\text {out }}$ ( $I_{\text {in }}$ and $I_{\text {out }}$ are the intensities of the input and output light) in the $n$-type germanium samples as a dependence on the $\mathrm{I}_{\text {in }}$ for two orientations of the light wave electric field in the crystal $\mathbf{E} \|(001)$ and $\mathbf{E} \|(111)$. The samples were cut from the germanium crystals in which the birefringence and FWI induced by the $\mathrm{CO}_{2}$ laser radiation were observed earlier. They have a shape of plane-parallel plates, two plates of which coincided with the (110) crystallographic plane of germanium. These surfaces were provided with antireflecting coatings. The linearly polarized light propagated along the [110] axis of the sample. The sample thickness in this direction was $0.5 \mathrm{~cm}$.

The source of IR radiation was either the pulsed periodic $\mathrm{CO}_{2}$ laser with the longitudinal electric discharge and Q-switching or the TEA-CO $\mathrm{CO}_{2}$ laser operating in the single pulse regime. The output pulse power was $10 \mathrm{~kW}$ for the first laser and $1 \mathrm{MW}$ for the second one. The radiation pulse durations was about 300 and $100 \mathrm{~ns}$, respectively. The pyroelectric detectors with the time resolution better than 3 ns measured an intensity of input and output light. A laser beam was focused on the sample surface by the $\mathrm{BaF}_{2}$ lens. The light intensity was varied from 1 to about $30 \mathrm{MW} / \mathrm{cm}^{2}$ by the calibrated $\mathrm{CaF}_{2}$ filters and/or by varying the distance between the lens and the sample. The measurements were carried out at the temperatures 300 and $77 \mathrm{~K}$. Since the light intensity after passing through a crystal has changed slightly, we calculated the absorption coefficient from the measured transmittance using the Bouguer formula. An error did not exceed $2 \%$. 
Fig. 1 shows the dependence of the absorption coefficient $\mathrm{K}$ of the studied $n$-Ge crystals on the intensity of the incident IR radiation of the $\mathrm{CO}_{2}$ laser $I_{\text {in }}$ with $10.6-\mu \mathrm{m}$ wavelength. The value of $\mathrm{K}$ is normalized to its magnitude at the low intensity $K_{0}\left(I \approx 10 \mathrm{~kW} / \mathrm{cm}^{2}\right)$. It is seen that, at the crystal temperature $300 \mathrm{~K}$ and the low intensities of light $\left(I \leq 12 \mathrm{MW} / \mathrm{cm}^{2}\right)$, the absorption coefficients are essentially equal for both field orientations of a light wave under study $(\mathbf{E} \|(111)$ and $\mathbf{E} \|(001))$. However, with the high intensities of light $I \geq 12 \mathrm{MW} / \mathrm{cm}^{2}$, the value of $K$ for $\mathbf{E} \|(001)$ becomes larger than that for $\mathbf{E} \|(111)$. At the crystal temperature $77 \mathrm{~K}$, this difference is observed for all the light intensities under study. Along with the dependence of the absorption coefficient on the electric field orientation of a light wave one observes also its increase with the light intensity growth. This is indicative of the fact that the absorption of light by free carriers in germanium is anisotropic and non-linear at the IR light intensities under consideration.

An increase of the absorption coefficient when $\mathbf{E} \|(111)$ and $\mathbf{E} \|(001)$ is different. With increasing the
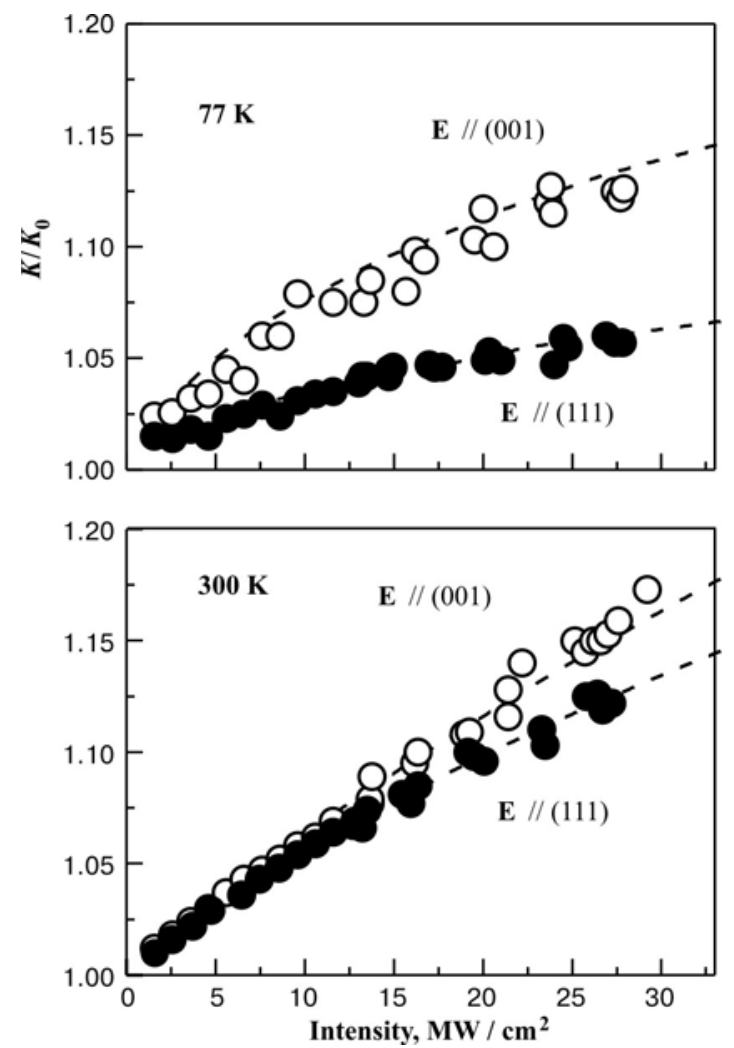

Fig. 1. The coefficient of light absorption by free electrons in Ge as a function of the IR light intensity for the light polarization vector oriented along the (100) and (111) crystallographic axes. The coefficient is normalized to the value corresponding to a low intensity $I=10 \mathrm{~kW} / \mathrm{cm}^{2}$. Circles - experiment, dashed lines - calculations. The carrier concentration: $N=510{ }^{16} \mathrm{~cm}^{-3}$. The light wavelength $\lambda=10.6 \mu \mathrm{m}$. light intensity from 2 to $29 \mathrm{MW} / \mathrm{cm}^{2}$ at $300 \mathrm{~K}$ it equals 12 and $15 \%$, respectively, it equals 4 and $11 \%$ while $77 \mathrm{~K}$. As a consequence, the anisotropy of absorption coefficients $\left(K_{(001)}-K_{(111)}\right)$ increases with a growth of the light intensity. At the maximal intensity $I_{\text {in }} \approx 29 \mathrm{MW} / \mathrm{cm}^{2}$, it equals to about $3 \%$ at $300 \mathrm{~K}$. With lowering the crystal temperature the anisotropy increases, and at $77 \mathrm{~K}$ for the same light intensity it is already about $7 \%$, almost 2.3 times higher than at $300 \mathrm{~K}$.

Let us consider the mechanism of these phenomena. In germanium for $\mathbf{E} \|(001)$, an intervalley redistribution of electrons is absent because the valleys are oriented symmetrically in regard to $\mathbf{E}$ and carriers in them are heated up equally [7]. The heating rate increases with a growth of the light intensity. Since with growing the average electron energy (the electron temperature) the electron scattering changes, the light absorption depends on its intensity. For the light with $\mathbf{E} \|(111)$, the heating electrons leads to their transition from three valleys of the $(\overline{1} 11)$ type into the valley of (111) type. For this reason, the effective mass of carriers along the $\mathbf{E}$ direction changes. Along with the changes in their scattering, this causes a non-linearity of light absorption. Because of the intervalley redistribution, the light absorption by electrons becomes also anisotropic.

Let us calculate the absorption coefficient of the $\mathrm{CO}_{2}$ laser IR radiation by free electrons in Ge crystals, taking into account the heating electrons in the light wave field and the intervalley redistribution. We showed earlier [8] that, at the temperatures 300 and $77 \mathrm{~K}$, and under conditions of equilibrium between free carriers and a crystal lattice, in Ge crystals the light absorption by these carriers at the wavelength $10.6 \mu \mathrm{m}$ is mainly connected with scattering the carriers by the acoustic and optical phonons. The part of light absorption coefficient related with the impurity scattering equals only $1.5 \%$ of the total absorption coefficient at $300 \mathrm{~K}$ and $6.5 \%$ at $77 \mathrm{~K}$. In $[8,9]$, obtained was the expression for the coefficient of light absorption by free electrons in the multivalley semiconductors through these scattering mechanisms for the case when the electron and lattice temperatures differ from each other. For scattering by the acoustic phonons it is written as

$$
\begin{aligned}
& K_{\mathrm{ac}}=\sum_{i} \frac{16 \sqrt{\pi}}{3 \sqrt{\varepsilon}} \frac{e^{2}}{c \hbar} \frac{N_{i} k T_{e}^{(i)}}{\gamma_{i}^{1 / 2} \omega^{3}} \cdot\left(1-e^{-Z_{i}}\right) \times \\
& \times \frac{1}{m_{t} \tau_{t}(T)}\left(\sin ^{2} \Theta_{i}+\alpha^{-1} \frac{\tau_{t}(T)}{\tau_{l}(T)} \cos ^{2} \Theta_{i}\right) \cdot \Psi_{\mathrm{ac}}\left(Z_{i}\right),
\end{aligned}
$$

$$
\begin{aligned}
& \Psi_{\mathrm{ac}}\left(Z_{i}\right)=Z_{i} \cdot \int_{0}^{\infty} x^{1 / 2}\left(x+2 Z_{i}\right)^{1 / 2} e^{-x} d x \\
& ++\int_{0}^{\infty} x^{3 / 2}\left(x+2 Z_{i}\right)^{1 / 2} e^{-x} d x
\end{aligned}
$$


where $\omega$ is the light frequency; $\theta_{i}$ is the angle between the axis of rotation of the $i$-th valley and the light polarization vector $\mathbf{E} ; N_{i}$ and $T_{e}^{(i)}$ are the electron concentration and temperature in the valleys; $\gamma_{i}=T / T_{e}^{(i)}, \quad Z_{i}=\hbar \omega / k T_{e}^{(i)}, \quad \tau_{l}(T)$ and $\tau_{t}(T)$ are, respectively, the longitudinal and transverse components of the relaxation time tensor for electrons with the energy $k T$ when scattering by the acoustic phonons takes place [10].

The light absorption coefficient when one deals with the scattering electrons by non-polar optical phonons equals to [8]

$$
\begin{aligned}
& K_{\mathrm{opt}}=\sum_{i} B_{i} \times \\
& \times \int_{0}^{\infty}\left\{\begin{array}{l}
N_{0}\left[x^{3 / 2}(x+S+Z)^{1 / 2}+x^{1 / 2}(x+S+Z)^{3 / 2}\right]+ \\
\left(N_{0}+1\right)\left[x^{3 / 2}(x+S-Z)^{1 / 2}+x^{1 / 2}(x+S-Z)^{3 / 2}\right]
\end{array}\right\} \times \\
& \times e^{-\gamma_{i} x} d x+
\end{aligned}
$$

$$
+\sum_{i} B_{i} e^{S \gamma_{i}} \times
$$$$
\times \int_{0}^{\infty}\left\{\begin{array}{l}
N_{0}\left[x^{3 / 2}(x-S+Z)^{1 / 2}+x^{1 / 2}(x-S+Z)^{3 / 2}\right]+ \\
\left(N_{0}+1\right)\left[x^{3 / 2}(x-S-Z)^{1 / 2}+x^{1 / 2}(x-S-Z)^{3 / 2}\right.
\end{array}\right\} \times
$$

$\times e^{-\gamma_{i} x} d x$,

where we use the following designation

$$
\begin{aligned}
& B_{i}=\frac{2 \sqrt{2}}{3 \pi^{1 / 2}} \frac{e^{2} D_{\mathrm{opt}}^{2} \sqrt{m_{l}}}{\rho \hbar^{4} c n \omega_{0}} \frac{(k T)^{3 / 2} \gamma_{i}^{3 / 2}}{\omega^{3}} \times \\
& \times\left(\sin ^{2} \theta_{i}+\frac{m_{t}}{m_{l}} \cos ^{2} \theta_{i}\right) \cdot N_{i}
\end{aligned}
$$

Here $D_{\mathrm{opt}}^{2}$ is the constant of interaction with the optical phonons, $N_{0}$ and $\omega_{0}$ are the number and frequency of phonons, $n$ is the refraction index, $S=\hbar \omega_{0} / k T$.

The optical absorption coefficient equals to the sum of the coefficients of light absorption by electrons in separate valleys, which depend on the light wave field orientation in regard to the valley axes, the carrier concentration in this valley and their temperature $T_{i}$. We find the electron temperature in valleys, as usual, from the balance of the powers added to the electron subsystem at the light absorption and that lost due to interaction of electrons with phonons and due to interelectron collisions.

Multiplying the coefficient of light absorption caused by electrons in one valley by the electromagnetic field energy flux incident on the semiconductor, we obtain the power transferred to the electron subsystem from a light wave. In numerous works, reported are the expressions for the average rate of energy losses due to the electrons scattering by the acoustic and optical phonons. We used such expressions in the form given in [11]. The energy exchange between electrons of different valleys at their collisions was calculated by the formulae presented in [10]. The constants, that characterize the mechanisms of electron scattering, the anisotropy parameter of scattering by the acoustic phonons, the optical phonon frequencies, and germanium band parameters were taken the same as from [7].

The electron concentration in the valleys was calculated from the equation of balance of the particle number. The redistribution of the "hot" electrons among valleys is assumed to occur in consequence of their scattering by the intervalley phonons. In this case, the equation of particle balance is written as follows [7]:

$$
\begin{aligned}
& N_{i} \gamma_{i}^{3 / 2}\left[e^{Z_{M}\left(1-\gamma_{i}\right)}+1\right] \int_{0}^{\infty} x^{1 / 2}\left(x+Z_{M}\right)^{1 / 2} e^{-\gamma_{i} x} d x= \\
& N_{j} \gamma_{j}^{3 / 2}\left[e^{Z_{M}\left(1-\gamma_{j}\right)}+1\right] \int_{0}^{\infty} x^{1 / 2}\left(x+Z_{M}\right)^{1 / 2} e^{-\gamma_{j} x} d x,
\end{aligned}
$$

where $Z_{M}=\hbar \omega_{M} / k T \quad\left(\omega_{M}\right.$ is the intervalley phonon frequency).

Fig. 1 presents the results of calculations for the absorption coefficient of the $\mathrm{CO}_{2}$ laser radiation by free electrons in the $n$-Ge crystals studied as dependent on the light intensity. It is seen from this figure that the calculated values of the absorption coefficient, and its behaviour with increasing the IR light intensity shows good agreement with the experimental data for both orientations of the light polarization in the crystal. This is indicative of the fact that, in the case of $n-\mathrm{Ge}$, the anisotropy and non-linearity of the light absorption by free electrons are caused by heating the carriers in the light wave field and their consequent redistribution among equivalent valleys.

Let us consider the influence only of heating electrons on the light absorption. It corresponds to the field orientation of a light wave along the (001) axis in germanium, when, as already mentioned above, the electron temperatures in all valleys and, consequently, the valley populations are equal. As following from (1) and (2), the dependence of the absorption coefficient on the electron temperature is weaker in the quantum case $\left(\hbar \omega>>k T_{e}\right)$ as compared to the classic one $\left(\hbar \omega<<k T_{e}\right)^{1}$. The calculated dependences of the absorption coefficient on the electron temperature in $n$ Ge at the lattice temperatures 300 and $77 \mathrm{~K}$ for the $\mathrm{CO}_{2}$ laser radiation with the polarization orientation coinciding with the (001) axis of the crystal are shown in

\footnotetext{
${ }^{1}$ For absorption with participation of the optical phonons one should take into account also relationship between $\hbar \omega_{0}$ and $k T_{e}$
} 


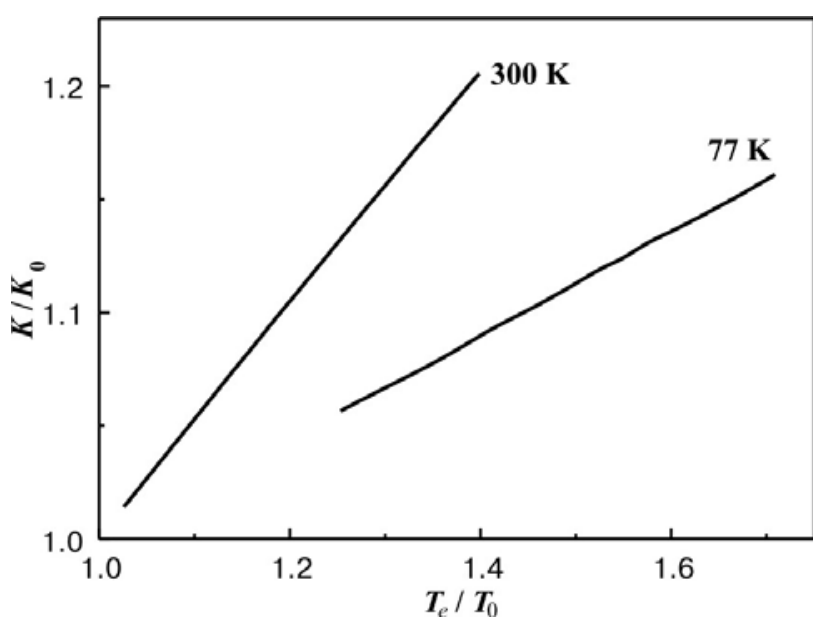

Fig. 2. Calculated dependence of the normalized absorption coefficient of the IR light with the wavelength $\lambda=10.6 \mu \mathrm{m}$ by free electrons in $n$-Ge on the reduced electron temperature. $N=5 \cdot 10^{16} \mathrm{~cm}^{-3}$.

Fig. 2. It is seen that, at both temperatures, the light absorption coefficient increases with growth of heating the electrons. At equal variations of the electron temperature, this increase is larger at $300 \mathrm{~K}$ as compared to $77 \mathrm{~K}$, in accordance with experimental results.

The quantum energy of the $\mathrm{CO}_{2}$ laser used in our experiments is about $1400 \mathrm{~K}$ while for the light intensities under study the electron temperature does not exceed 400 and $120 \mathrm{~K}$ at the lattice temperatures of 300 and $77 \mathrm{~K}$, respectively. This demonstrates that, in the experiment, realized is an intermediate situation that corresponds rather to the classic case at $300 \mathrm{~K}$ than to the quantum one at $77 \mathrm{~K}$. It seems to be connected with different influence of heating electron on the light absorption by electrons at these crystal temperatures.

The authors are greatly indebted to Prof. O.G. Sarbey for fruitful discussion of the results.

\section{References}

1. A.K. Walton, Infrared modulation and energy band parameters in multivalley semiconductors through uniaxial stress dependence of free carrier contribution to optical constants // Phys. status solidi (b) 43, N1, p. 379-386 (1971).

2. K. Seeger, H. Vana, Hot-carrier infrared absorption in n-type germanium // Ibid. 96, N 2, p. 605-610 (1979).

3. P.M. Tomchuk, A.A. Chumak, Nonlinear propagation of infrared radiation in many-valley semiconductors // Sov. Phys. Semicond. 19, p. 46-52 (1985).

4. V.N. Poroshin, O.G. Sarbey, V.M. Vasetskii, Nonlinear optical phenomena related to "hot" electrons in multivalley semiconductors // Ukr. Fiz. Zhurn. 44, N 1-2, p. 60-65 (1999) (in Ukrainian).

5. V.M. Vasetskii, V.N. Poroshin, V.A. Ignatenko, Degenerate four-wave mixing in $\mathrm{n}-\mathrm{Ge}$ due to intervalley redistribution of hot electrons // Semiconductor Physics, Quantum Electronics \& Optoelectronics 4, N 4, p. 260-263 (2001).

6. V.L. Malevich, Anisotropy of the nonlinear free carrier absorption in n-Ge // Fiz. Tekh. Poluprovodn. 16, N 11, p. 2035 -2037 (1982) (in Russian).

7. M. Asche, Z.S. Gribnikov, V.V. Mitin, and O.G. Sarbey, Hot electrons in many-valley semiconductors, Naukova Dumka, Kiev (1977) (in Russian).

8. V.N. Poroshin, O.G. Sarbey, The role of optical phonons in electrons heating by IR radiation in Ge // Semiconductor Physics, Quantum Electronics \& Optoelectronics 8, N 2, p. 1-3 (2005).

9. P.M. Tomchuk, Peculiarities of the light absorption and light emission by free electrons in multivalley semiconductors // Ukr. Fiz. Zhurn. 49, N 7, p. 681690 (2004).

10. I.M. Dykman, P.M. Tomchuk, The transport phenomena and fluctuations in semiconductors, Naukova Dumka, Kiev (1981)(in Russian).

11. M. Asche, O.G. Sarbey, Electric conductivity of hot carriers in Si and Ge // Phys. status solidi 33, p. 9-57 (1969). 